REVISTA DE DERECHO UNED, núm. 6, 2010

\title{
MITOS Y REALIDADES DE LA EDUCACIÓN VIRTUAL (E-LEARNING) DE CARÁCTER ASINCRÓNICO. SU APLICACIÓN EN CURSOS DE DERECHO Y CIENCIA POLÍTICA
}

\author{
JuAn Jacobo NúÑEZ MartíneZ \\ UNED
}

Resumen: A falta de unos estándares claros de referencia sobre la educación virtual que nos orienten respecto al grado de calidad de este tipo de formación, hemos realizado un estudio que tiene en su base la comparación de masteres de muy similares características a fin de confrontarlos sobre las principales ventajas o inconvenientes que la bibliografía ha señalado.

El resultado obtenido nos ha indicado que se han cumplido todos los parámetros propuestos, así como que también se han detectado otros nuevos problemas que consideramos extrapolables a o otros cursos on line de la misma área temática y que su resolución podría suponer ventajas competitivas a futuros cursos.

Palabras clave: educación virtual, formación, evaluación, asincrónica, ciencias sociales y jurídicas.

Abstract: In absence of clear reference standards regarding online virtual education that can guide us with respect to the level of quality of this type of formation, we have performed a study based on the comparison of masters of similar characteristics in order to compare them in relation to the principle advantages or inconveniences that the bibliography has shown.

The outcome reveals that all the proposed parameters have been met, and in fact, other new problems have been detected which we consider could be applied to other online courses of the same the- 
matic area and that it's resolution could suppose competitive advantages to future courses.

Key words: virtual education, formation, asincronic evaluation, social sciences and judicial sciences.

Sumario: Introducción.-1. Desarrollo.-1.1. Másters objeto de estudio.-1.2. Procedimiento y metodología.-1.3. Limitaciones de la investigación.-2. Resultados.-2.1. Perfil de los alumnos objeto del estudio.-2.2. Pone a disposición del alumno un amplio volumen de información.-2.3. Flexibilidad horaria y espacial.-2.4. Ofrece diferentes herramientas de comunicación asincrónica y sincrónica.-2.5. Favorece la formación multimedia.-2.6. Ahorro en costes.-2.7. Relación entre alumno y profesor.-2.7.1. Relaciones con los profesores.-2.7.2. Relación con los coordinadores.-3. Conclusiones.-4. Bibliografía.

\section{INTRODUCCIÓN}

Si bien la educación a distancia tiene su origen en el siglo XVIII; no es hasta finales de la década sesenta del siglo XX y como resultado de una mejora en las telecomunicaciones cuando este sistema se implanta en la educación superior, surgiendo así las primeras universidades totalmente a distancia ${ }^{1}$.

No obstante, todas las fuentes utilizadas coinciden en que su expansión no se produce hasta la segunda mitad de los años noventa, como consecuencia de dos aspectos interrelacionados entre sí como son la generalización del acceso a Internet en los países desarrollados y la aparición de la denominada sociedad del conocimiento.

Por este motivo, a día de hoy, la formación on line es considerada como sinónimo de formación a distancia, puesto que es la herramienta idónea que permite a los trabajadores obtener una enseñanza actualizada y especializada pero a la vez compatible con su vida profesional.

La formación on line ofrece ventajas que la hacen muy atractiva y que se relacionarían con los siguientes aspectos: un ahorro en costes de realización frente a la enseñanza presencial entre un 30\% y un

1 Durante los años sesenta y setenta del siglo pasado se crean las siguientes universidades: Open University en Gran Bretaña ( 1969); Universidad de Phoenix en Estados Unidos (1976), Universidad Nacional de Educación a Distancia en España (1974), Universidad a Distancia de Hagen en Alemania ( 1975). 
60\% menos (Castells, 1996), una falta clara de regulación que ha permitido la aparición de plataformas web de toda índole, ofertando una gran variedad de cursos académicos ${ }^{2}$, y grandes posibilidades de expansión debido tanto a la globalización como a la flexibilidad que aporta (Altvater, 2000; Bello, 2003).

Por ello no es de extrañar que tanto instituciones públicas ${ }^{3}$ como privadas $^{4}$ hayan adoptado este nuevo modelo formativo como potencial fuente de ingreso y expansión (John Chamber, 2001) al tratarse de un mercado de futuro ${ }^{5}$.

Si bien nadie discute las ventajas de la educación virtual o e-learning (Ruhland \& Brewer, 2001; Barrón, 2004; Cabrero y Duarte, 1999; Barbera, 2001); en los últimos años han surgido voces cada vez más críticas que desde distintos sectores cuestionan este tipo de formación, advirtiendo de las carencias que todavía puede tener un formato relativamente nuevo.

No debemos olvidar que el entorno de Internet es limitado; y si bien permite realizar la formación desde cualquier lugar que disponga de conexión; estos sistemas pueden reducir el proceso de transmisión del saber a la simple producción, distribución y aplicación del contenido informático (Bates, 2001), sin tener en consideración cómo están diseñados y producidos los materiales ni otros aspectos esenciales de la formación como pueden ser la pedagógica necesaria. Como indica Dirk Schneckenberg (2004), Intenet no sabe sustituir el contacto humano y directo entre el profesor y los estudiantes. Por ello, solamente si tenemos en cuenta las limitaciones de un medio como Internet, se puede evitar crear falsas esperanzas.

2 Por ello no es de extrañar la aparición de términos como digital diploma mill para referirse a la expedición de títulos académicos, ya que se ofertan una gran variedad de cursos que carecen de sustento institucional y pedagógico.

3 Cabe destacar como ejemplos a la propia Unión Europea con su iniciativa para el fomento de la formación e-learning a través de la plataforma European elearning Portal en el año 2001 o las universidades españolas de carácter presencial con la creación de campos virtuales comos CAI, EURORED y otras para la aplicación de la metodología e-learning.

${ }^{4}$ En el ámbito privado en empresas como know-how o Big Money para la explotación del sector de la educación.

5 A modo de ejemplo indicaremos que tal y como recoge el informe «El estado del arte de la formación» (ADECO: 2009) durante el año 2008 el e-learning experimentó un aumento de un 30\% con respecto al año anterior.

Estos datos, con ser importantes, no son comparables con la subida experimentada durante el periodo comprendido entre los años 2004 y 2005 donde se produjo un incremento de un 70\% (Carlos Cánepa 2005). 
Gallego y Martínez (2008) indican que la retroalimentación puede ser muy lenta, que es más difícil la rectificación de errores en los materiales, evaluaciones, etc, y existen más abandonos que en la enseñanza presencial.

Un sondeo europeo llevado a cabo por CEDEFOP en el año 2002 a alumnos que habían realizado cursosvirtuales, indicaba que un $61 \%$ de los encuestados valoraban de forma negativa la calidad del aprendizaje electrónico obtenido calificándolo de malo o insuficiente, mientras que únicamente un 5\% lo consideraba como muy bueno y un $1 \%$ como excelente.

En este sentido Blázquez y Alonso (2005) indican que la gran mayoría de los alumnos se inclinan por el método de la educación presencial porque es más directo y en él se pueden aclarar mejor las dudas.

Debemos recordar que los cursos e-learning se han desarrollado con mayor intensidad dentro del ámbito de las ciencias sociales y jurídicas que en otro campo como pudieran ser las ciencias físicas o las ingenierías, ya que en el primer campo se nutre de contenidos mucho más teóricos que prácticos, y por tanto se adaptan mejor a la metodología de este tipo de cursos.

El objetivo de este trabajo es comprobar, a través de la experiencia adquirida con dos Másters de similares características y pertenecientes ambos al ámbito de la Ciencia Política y el Derecho, si las supuestas ventajas o problemas que ofrece la enseñanza e-learning se han producido en ambos casos.

Para la realización de esta investigación se han utilizado herramientas de primera mano recogidos en las plataformas web, los correos electrónicos de alumnos, coordinadores, tutores, las evaluaciones, etc.. Así como opiniones y encuestas realizadas tanto a los alumnos como a los trabajadores del Máster.

Se ha intentado evitar las particularidades de las maestrías, buscando únicamente aquellos aspectos que pudieran ser extrapolares a otros cursos on line de la misma área temática.

\section{DESARROLLO}

Desde el comienzo de la formación virtual uno de sus campos de acción han sido los Másters y cursos relacionados con la ciencia jurídica y la gestión de administración pública o privada. 
Según la revisión bibliográfica consultada, las características más destacables que se ha observado en la realización de estos cursos o másters han sido las siguientes:

- Alumno motivado e innovador.

- Pone a disposición del alumno un amplio volumen de información.

- Flexibilidad horaria y espacial $(24 \times 7 \times 365)^{6}$.

- Ofrece diferentes herramientas de comunicación sincrónica y asincrónica.

- Favorece la formación multimedia.

- Ahorra costes de desplazamiento.

- Favorece la interactividad en diferentes ámbitos con el profesor y los alumnos.

Sobre dichas premisas, hemos realizado el estudio comparativo a fin de comprobar si las mismas se han cumplido también en las maestrías objeto de estudio.

\subsection{Másters objeto de estudio}

Los Másters analizados para la realización de este trabajo han sido los siguientes:

II Máster a Distancia de Gerencia Pública para Directivos Iberoamericanos (MADGP), realizado por la Universidad Nacional de Educación a Distancia (UNED) y destinado a altos cargos públicos iberoamericanos. Dio comienzo en el año 2005 y desde dicha fecha se han realizado las ediciones de forma continuada en los siguientes países: República de Colombia y la República Bolivariana de Venezuela. Este Máster es continuación de otro anterior que bajo el mismo nombre tuvo una primera edición en el año 2001 con cursos en Bolivia, Perú y la República Dominicana.

Máster en Gestión y Análisis de Políticas Públicas (MEGAP), realizado por la Universidad Carlos III conjuntamente con la Fundación Internacional y para Iberoamérica de Administración y Políticas Públicas (FIIAPP), destinado exclusivamente a altos cargos de la Administración Pública Iberoamericana dando comienzo en marzo de 2007 con la siguiente edición: Perú.

\footnotetext{
624 horas al día, por siete días a la semana por 365 días al año.
} 
Similitudes entre ambos másters:

- Ambos Másters se encuentran conformados por un mismo segmento de alumnado (altos cargos de la Administración Pública Iberoamericana)

- Ambos Másters tiene una duración total de 24 meses con una equivalencia de 600 horas lectivas (equivalentes a 60 créditos lectivos) y una estructura de desarrollo compuesto a través de módulos de carácter temático.

- El temario impartido en los dos Másters es similar en un 80\%, puesto que en ambos se abordan los mismos contenidos relacionados con el desarrollo de las políticas públicas.

- La calidad del profesorado que imparte los módulos es análoga, puesto que en ambos casos se trata de catedráticos y titulares de universidad de las mismas áreas de conocimiento, así como de altos cargos de la Administración Pública española, coincidiendo, en algunos casos, que un mismo profesor participe en ambas maestrías.

- El precio por la realización del Máster también es coincidente ${ }^{7}$, existiendo en ambos un alto porcentaje de alumnos becados al $100 \%$.

- La estructura organizativa es igual, ya que en ambos casos se encuentran dirigidos por un Director Académico un coordinador y un reducido equipo para la gestión administrativa de los mismos.

- Los dos Másters se encuentran alojados en páginas web de similares características (plataforma Windows NT Server 4.0 y un software de servicio de Internet - Microsoft Information Server o Apache).

- Los dos Másters son de carácter asincrónico y la metodología empleada es la propia de la enseñanza a distancia utilizando las innovaciones de la educación virtual: módulos alojados en la propia página web que pueden ser descargados por los alumnos, correo electrónico, foro de discusión, biblioteca virtual, lista de distribución, enlaces de interés.

7 El precio por la realización del MEGAP fue de 2.500 US $\$$ y en el caso del MADGP su precio fue de 3.000 US\$ 
Mitos Y REALIDADES DE LA EDUCACIÓN VIRTUAL (E-LEARNING)...

- En ambos casos el título que se obtiene es un título propio de la Universidad.

Diferencias entre ambos Másters:

- El MADGP es gestionado directamente por la UNED, mientras que el MEGAP es gestionado por una institución ajena a la universidad ( FIIAPP).

- La estructura académica del Máster difiere de un Máster a otro, ya que el MADGP consta de tres grandes áreas temáticas (Teoría del Estado, Economía y Gestión presupuestaria y Gerencia Pública y Recursos humanos) que engloban un total de 17 módulos, todos ellos de carácter obligatorio. Realizándose una evaluación a la finalización de cada área temática (cada seis meses).

- La estructura académica del MEGAP consta de un total de 15 módulos a realizar por el alumno, de los cuales 6 son de carácter obligatorio, 7 de carácter optativo a elegir entre una oferta de 12 módulos y 2 obligatorios específicos territoriales que variarán en función del país, realizándose una evaluación de carácter mensual, coincidiendo con la finalización de cada módulo.

\subsection{Procedimiento y metodología}

La obtención de la información se ha realizado a través de diversos instrumentos que han permitido la triangulación de los resultados para esta investigación.

En primer lugar se ha realizado un cuestionario a los estudiantes de ambos másters de carácter obligatorio y a fin de obtener la mayor sinceridad posible en sus contestaciones, este se realizó de forma anónima coincidiendo con la realización de las actividades presenciales realizadas en dichos países.

En segundo lugar se ha utilizado la información obtenida durante todo el desarrollo de los Másters a través tanto de las preguntas enviadas por los alumnos a los coordinadores y profesores y sus posteriores respuestas, así como toda la información volcada en las páginas web ${ }^{8}$ durante el desarrollo de las maestrías.

\footnotetext{
${ }^{8}$ Entendido como preguntas de foro, Chat, entradas por parte de los alumnos en links, envío de exámenes por vía telemática, etc.
} 
En tercer lugar se han obtenido diversos datos de carácter demográfico a partir de las informaciones recogidas en los formularios de matrícula que han servido para contrastar los datos recogidos.

Por último, se ha obtenido información de carácter cualitativo realizado a través de entrevistas y comentarios a los coordinadores de los Másters.

\begin{tabular}{|l|c|c|}
\hline & MEGAP & MADGP \\
\hline N. ${ }^{\circ}$ de Alumnos & 101 & 97 \\
\hline N. ${ }^{\circ}$ de abandonos & 40 & 58 \\
\hline N. ${ }^{\circ}$ de Aprobados & 48 & 33 \\
\hline N. ${ }^{\circ}$ de Suspensos & 13 & 4 \\
\hline
\end{tabular}

\begin{tabular}{|l|c|c|}
\hline \multicolumn{1}{|c|}{ EDADES } & MEGAP & MADGP \\
\hline $25-36$ & 39 & 32 \\
\hline $37-45$ & 43 & 48 \\
\hline $46-55$ & 18 & 17 \\
\hline $56-65$ & 1 & 0 \\
\hline
\end{tabular}

\section{RATIOS COMPARATIVOS DE AMBOS MÁSTERS}

\begin{tabular}{|l|c|c|}
\hline \multicolumn{1}{|c|}{ EDADES } & MEGAP & MADGP \\
\hline Hombres & 73 & 61 \\
\hline Mujeres & 28 & 36 \\
\hline
\end{tabular}

\subsection{Limitaciones de la investigación}

Las principales limitaciones de nuestra investigación es que obviamente la población objeto de estudio es un colectivo no representativo del conjunto de los estudiantes de este tipo de cursos. Es por ello que no hemos pretendido obtener conclusiones que sean generalizables estadísticamente, sino realizar principalmente un análisis descriptivo y exploratorio que permita avanzar en el establecimiento de nuevas líneas de investigación.

\section{RESULTADOS}

\subsection{Perfil de los alumnos objeto del estudio}

Según la bibliografía consultada, el perfil de los alumnos de la maestría es coincidente con el de los cursos on-line; pues los estudios realizados (Dutton et al., 2002) indican que nos encontraríamos con estudiantes adultos, con una media de edad superior al de los estudiantes off-line, pero con una gran diversidad de edades. En el caso del MADGP las edades oscilan en una orquilla desde los 25 
Mitos y REALIDADES DE LA EDUCACIÓN VIRTUAL (E-LEARNING)...

años a los 55; y en le caso del MEGAP la orquilla correspondería a edades comprendidas entre los 25 a los 65 años.

En cuanto al perfil motivacional de los alumnos on-line (Dutton et al., 2002; Chiencher, 2004), se trataría de un alumno consciente de su necesidad formativa, laboralmente activos y que deben restringir su formación a los ajustes de los horarios tradicionales.

Según se desprende de las respuestas en las hojas de inscripción a la pregunta ¿cuál es la razón por la cuál quiere realizar este Máster? Dicho perfil también es coincidente, ya que un $90 \%$ de los alumnos de ambas maestrías indicaron como factor motivacional: adquirir nuevas herramientas de gestión que les permitiesen una mejora en su desarrollo profesional.

Respecto a su situación laboral, recordemos que en ambos casos para participar en los Másters, era condición indispensable trabajar en el sector público.

En este estudio se han detectado también otras dos motivaciones coincidentes en ambas maestrías y que serían las siguientes:

La primera se relacionaría con la política de becas seguida en ambos Másters, que en el caso del MEGAP cubrió a un total de 80 alumnos y en el caso del MADGP a un total de 37 alumnos.

En el caso del MEGAP las selección para las becas fue realizada por la contraparte local entre una gran demanda de postulantes.

En el caso del MADGP las becas fueron concedidas directamente por la UNED a petición de la contraparte local.

La política de becas en ambos casos fue realizada siguiendo criterios tradicionales de la enseñanza presencial ${ }^{9}$, aunque con algunos matices en el caso del MEGAP. Posteriormente se pudo comprobar que dicha política fue errónea; ya que el índice de abandonos (porcentualmente) fue muy superior en alumnos becados que en aquellos alumnos que pagaron la matrícula completa (en el caso del MADGP un 59\% de los abandonos se produjo en el caso de los alumnos becados y en el caso del MEGAP la cifra ascendió al 100\%, ya que ningún alumno de pago abandonó el Máster).

Consideramos que para evitar el alto índice de abandonos que se producen en la enseñanza on line, uno de los mayores factores críticos de este modelo formativo, a la hora de realizar la política de becas

9 Renta, discapacidad, familia numerosa, etc. 
se debería tomar en consideración otros aspectos en la selección como ya señaló en su día la Fundesco en 1998, indicando aquellas características concretas que debía reunir un alumno idóneo, y que distintos autores también han analizado, (Calero, 2006; Cánepa, 2005; Blázquez-Alonso, 2005) y que son una metodología especial, un aprendizaje autónomo, una capacidad de búsqueda, fuerza de voluntad... a fin de evitar el alto índice de deserciones.

La segunda motivación se referiría a la posibilidad de obtener un título académico emitido por una universidad sin la necesidad de desplazarse durante el periodo de formación a otro país.

Este hecho estaría constatado en el caso del MEGAP, pues el título español se emitía conjuntamente con el de otra universidad local; según se dedujo de los correos emitidos por los alumnos, un $88 \%$ de los mismos, centraban su interés en la obtención del título de la universidad española.

En el caso del MADGP el hecho fue más evidente aún, ya que en un principio se planteó la posibilidad de realizar conjuntamente el título con una institución del país; finalmente dicha opción no fue posible, ofreciendo a los alumnos la posibilidad de reintegrarles el importe de sus matrículas. Únicamente cinco alumnos optaron por la restitución del dinero dándose de baja en el Máster.

Este aspecto confirmaría que parte del éxito de los cursos online se debe a la posibilidad de poder obtener una titulación de cualquier parte del mundo sin tener la necesidad de desplazarse.

\subsection{Pone a disposición del alumno un amplio volumen de información}

Una de las grandes ventajas de los cursos e-learning consiste en la posibilidad de proveer de la información necesaria a los alumnos en el momento que la necesiten (just in time), pues (Jorba y Sanmartí, 1994) esta educación da acceso a diferentes fuentes de información que permiten al alumno profundizar en aquellos aspectos que le resulten de especial interés de forma sencilla a través de la propia plataforma web, potenciando la conectividad y la conexión interdisciplinar de las materias.

Esta situación aún es más patente en aquellos cursos o Másters que se circunscriben dentro del ámbito de la Ciencia política o el Derecho debido a la gran variedad de información existente en la red al 
Mitos y REALIDADES DE LA EDUCACiÓN VIRTUAL (E-LEARNING)...

respecto, así como de páginas web gubernamentales que suministran una gran cantidad datos o documentación sobre aspectos concretos en este campo.

Siguiendo las directrices no escritas sobre la enseñanza a distancia (en especial la relacionada con los de $3^{a}$ generación) en ambos máster se adjuntaron links y documentación específica de apoyo a los alumnos.

El resultado fue el siguiente:

En el MEGAP la información adicional se alojaba a través de dos links: un primer link contenía direcciones web de organismos oficiales de ámbito público y un segundo link denominado biblioteca que albergaba documentación específica sobre la gestión pública (revistas especializadas, paper work, libros, documentación de congresos...) Ambos link eran actualizados de forma regular.

En los primeros meses del MEGAP un 65\% de los alumnos entraban en ambos links de forma regular; cifra que fue descendiendo progresivamente hasta un $8 \%$ al final de la maestría en el link correspondiente a biblioteca y a un $0 \%$ en el link correspondiente a direcciones web.

En el caso MADGP la documentación adicional era suministrada a través de una lista de distribución en la que eran enviados enlaces web, documentos o noticias.

La comprobación de la lectura de esta documentación era realizada a través de los foros, pues se volcaban preguntas relacionadas con las materias enviadas. En los primeros meses se recibieron un total de 22 mensajes ( $21 \%$ de los alumnos), cifra que fue disminuyendo hasta las 6 entradas (6\% de los alumnos) al final de la maestría.

Las razones de este descenso son diversas, pero la información estudiada nos indica que pasada la novedad de los primeros meses, los alumnos comenzaron a economizar su tiempo, centrándose en aquella documentación (módulos) que les resultase útil para la realización de las evaluaciones. Esta hipótesis quedaría reforzada al comprobar que durante el desarrollo del Máster, cada vez eran más los alumnos que enviaban sus evaluaciones en la fecha límite o fuera de la misma, tal y como desarrollaremos en el siguiente apartado.

Otro motivo tal y como han aventurado a comentar distintos autores (Blázquez y Alonso, 2005; Calero, 2006; Koskinen, 1998 o Chickering y Ehrmann, 1996) puede deberse a la tendencia en los cursos on-line a introducir excesivo material adicional sin tener en cuenta 
que el nivel de necesidad de información de los alumnos es limitado; pues como apunta Simith (2006) una gran cantidad de información no resulta siempre fácil de asimilar. Tal y como indica Bates (2001), en muchas ocasiones se valora más la facilidad de volcar el material que el material en si mismo y la medición pedagógica que genere.

\subsection{Flexibilidad horaria y espacial}

Una de las grandes ventajas de la formación on-line es su flexibilidad horaria y temporal, permitiendo al alumno poder estudiar el material docente en el horario más conveniente y administrarse su propio ritmo de estudio, gracias a que como indica Barrón (2004) los alumnos tienen un plan de trabajo, unas guías de estudio y unos calendarios que les sirven de guía.

Según la comprobación de ambos másters, dicha flexibilidad no se ha producido debido a los siguientes motivos:

En referencia a la flexibilidad horaria indicar que en el caso MEGAP a la pregunta ¿en qué horario accedían al sistema? Un $25 \%$ indicó por la mañana, un $32 \%$ por la tarde y un $43 \%$ por la noche en sus domicilios particulares.

Entre aquellos alumnos que realizaban las entradas por la mañana $(57 \%)$ la razón esgrimida fueron los problemas derivados de la conexión a Internet, ya que debido a la cobertura o al peso de los materiales, tenían problemas para poder acceder desde sus domicilios particulares al tener una conexión más lenta; y por tanto bajan los materiales en horario laboral para posteriormente desarrollar su estudio en sus hogares.

En el caso del MADGP la situación indicó que un 28\% accedían al sistema por la mañana o por la tarde, mientras que un $72 \%$ lo efectuaban por la noche en sus domicilios particulares. La razón esgrimida para el turno mañana-noche fue la misma, aunque un $12 \%$ reconoció que el motivo residía en no tener conexión a Internet en sus propios hogares. Por dicho motivo suscribimos las palabras de Bonta (1998) cuando afirma que el hogar vuelve a ser el núcleo de la educación desde la introducción de las redes virtuales.

En referencia a la flexibilidad temporal, se ha comprobado que la formación on line es similar a la formación tradicional, y finalmente depende de la voluntad e interés del alumno por el estudio, ya que se tratan de Másters cerrados donde (Cabero J., 2006) se han trasladado 
Mitos y REALIDADES DE LA EDUCACiÓN VIRTUAL (E-LEARNING)...

los principios aplicados de la enseñanza presencial y que se resumen en una serie de aspectos poco flexibles con fechas de finalización e inicio, pero con la diferencia de que en la educación e-learning existe la posibilidad de acceso y consultas abiertas.

En el caso del MEGAP con evaluaciones obligatorias de carácter mensual enviadas a través de la propia página web, indicaremos que como media del curso, únicamente un 10\% de los alumnos entregaban sus evaluaciones entre 5 y 2 días antes de la fecha límite de entrega; un $60 \%$ el mismo día de la finalización del plazo y un 30\% pasado dicho plazo, aún sabiendo que la evaluación podía no ser admitida.

En el caso que una fecha límite de entrega coincidiese en viernes, la entrega de dichas evaluaciones (un 70\%) era realizado el lunes siguiente.

En el caso del MADGP aunque el sistema de evaluación difería del MEGAP (tres únicas evaluaciones a lo largo de la maestría) los resultados indicaron que un $15 \%$ enviaba sus evaluaciones antes del plazo establecido, un $60 \%$ en la fecha y plazos establecidos y un $25 \%$ pasada la fecha límite de entrega.

\subsection{Ofrece diferentes herramientas de comunicación asincrónica y sincrónica}

El modelo asincrónico tiene su base en el aprovechamiento máximo de los potenciales de los entornos virtuales de aprendizaje (EVA) que aportan fundamentalmente flexibilidad e interactividad y permiten la vinculación a una verdadera comunidad virtual de personas que aprenden ( Duart y Sangrá. 2000).

Esta comunicación permite al alumno y al profesor trabajar a su propio ritmo, reflexionar y analizar las cuestiones de forma pausada.

Pero la comunicación puramente asincrónica también tiene detractores (Blázquez y Alonso 2005) debido a la falta de espontaneidad que genera (comunicación fría) y a la pérdida de matices de la comunicación presencial que pueden llevar al alumno a la deserción por la falta de dinamismo existente en este modelo de comunicación, o a la percepción que los alumnos puedan tener sobre la calidad del Máster. Por este motivo muchos cursos están optando por el modelo blended learning o aprendizaje mezclado ${ }^{10}$.

\footnotetext{
${ }^{10}$ Mezcla actividades pedagógicos presenciales con actividades a distancia.
} 
Tanto en el MEGAP como en el caso del MADGP se optó por la comunicación asincrónica como única vía debido a las diferencias horarias y geográficas existentes entre la sede de los másters y la ubicación de los alumnos.

Este modelo de comunicación fue muy valorado por los alumnos, teniendo una aceptación de un 90\% en el caso del MEGAP y de un $98 \%$ en el MADGP, pues la entendían como la única comunicación posible para la realización del Máster.

En ambos Máster el correo electrónico fue la herramienta principal para realizar esta comunicación, siendo el tiempo de respuesta a los alumnos por parte de la coordinación de 12 horas como máximo, exceptuando fines de semana que era de 48 horas.

En el caso del MEGAP 76\% de los alumnos indicó como buena o muy buena la interacción con la coordinación del Máster, mientras un $24 \%$ la indicaba como mala o muy mala; a la pregunta del motivo, la respuesta en la mayoría de los casos radicaba en la falta de agilidad en la respuesta de los correos electrónicos.

En el caso del MADGP la interacción con los coordinadores fue peor valorada ya que un $45 \%$ la consideraron como buena o muy buena, mientras que un 55\% como mala o muy mala.

En función de las críticas vertidas y los problemas planteados por los alumnos, a continuación exponemos aquellas generadas en ambos Másters y que consideramos más significativas:

El principal problema que se detectó y que no hemos visto referenciado explícitamente por ninguno de los autores consultados, se relacionaría con la necesidad por parte de los alumnos de la resolución de problemas de forma inmediata.

Uno de los aspectos que más flujo de correos ha generado, tuvo su origen en problemas para el envío de evaluaciones o problemas web y que debido a las diferencias horarias eran imposibles solucionarlos de forma automática. La falta de costumbre de los alumnos habituados a una educación presencial caracterizada por el «just in time» ha provocado situaciones de ansiedad y frustración al no obtener esta inmediatez y olvidar los alumnos que se encontraban realizando un curso on line.

El segundo problema y que consideramos específico de los Máster y cursos tanto de la Ciencia Política como del Derecho se relacionarían con la materia tratada. 
Mitos y REALIDADES DE LA EDUCACióN VIRTUAL (E-LEARNING)...

Existen dificultades por parte de los alumnos para comprender determinados conceptos jurídicos como pueden ser Estatutos de Autonomía, Leyes Orgánicas, etc., que necesitan explicaciones extensas que muchas veces el correo electrónico no puede subsanar.

Por este motivo se tuvo que recurrir a la comunicación telefónica a fin de que el alumno pudiese intercomunicarse con el tutor e intercambiar comentarios de una manera más satisfactoria.

El tercer problema tendría su origen en la inseguridad que generan los cursos on line en los alumnos ante la posibilidad de que sus contribuciones no sean recibidas.

Si bien en ambos másters se garantizaba dicho aspecto, el alumnado todavía desconfía en este sistema, tal y como se comprobó a través del envío masivo de correos electrónicos por parte de los alumnos sobre una misma cuestión a todas las direcciones web del Máster.

Debemos resaltar que en ambos máster no se ha producido una de las críticas que se suelen realizar a este tipo de formación y que sería la perdida de la interacción presencial que retarde la construcción de la identidad de grupo y la cohesión.

Puede deberse a que todos los participantes pertenecían a la Administración Pública o como consecuencia de los canales de comunicación existentes, los grupos estuvieron muy estructurados, existiendo incluso delegados de alumnos en ambos masters.

\subsection{Favorece la formación multimedia}

La American Society of Training and Development indicó que el aprendizaje que tiene con su base en la web es un aprendizaje con fundamento en ordenadores, aulas virtuales y colaboración digital. Por ello es una excelente herramienta para ayudar a los usuarios pues no solo aprenden nuevos conceptos, sino también afianzan habilidades a través de una inmersión práctica.

A través del Máster se comprobó que todos los alumnos mejoraron su nivel informático; no obstante dicha mejora se produjo en detrimento de la propia maestría.

En ambos Másters se entregaron antes de su comienzo guías didácticas sobre el funcionamiento de la plataforma web ${ }^{11}$, así como

11 Guías volcadas también en las propias páginas web. 
también se realizaron reuniones presenciales previas a fin de explicar su funcionamiento.

Una vez que ambas maestrías fueron puestas en funcionamiento, se detectaron los siguientes problemas:

Un primer problema tuvo su origen en el descubrimiento de que un $12 \%$ de los alumnos del MEGAP y un $20 \%$ de las alumnos del MADGP desconocían nociones básicas de la web, tales como ¿qué es un Chat?, ¿qué es un foro?, ¿o qué es el sistema adobe?.

Un segundo problema consistió en que a pesar de las explicaciones realizadas sobre el funcionamiento de la página web, un alto porcentaje de los alumnos tuvo problemas de comprensión sobre la mecánica de la página, aunque estas dificultades fueron remitiendo en la medida que los alumnos fueron familiarizándose pasados los primeros meses.

Pero posiblemente el principal problema de la maestría en el aspecto tecnológico tiene su origen en la conexión a Internet y la capacidad de las computadoras de los alumnos. Este problema se agravaba con aquellos alumnos que estudiaban la maestría en zonas interiores de los países donde las comunicaciones no son todo lo deseable que deberían ser. Así, Mendez-Estarada y Monge Najera (2006) han señalado este motivo como el verdadero «talón de Aquiles» de los Máster on line con determinados países.

Estos problemas provocaron un sentimiento de frustración entre el alumnado, tal y como se corroboró con la realización de las primeras encuestas, confirmando lo ya indicado por algunos autores como Davie (1998), Cornell (1999) o Zirkin y Sumler (1995) por poner algunos ejemplos, que indican la relación directa entre la motivación y el aprendizaje como consecuencia de los problemas de interactividad en los cursos on-line.

Un $80 \%$ del MEGAP indicó haber experimentado problemas con el sistema en la plataforma virtual apuntando como principales problemas por orden de importancia: 1) que no siempre se abría la página; 2) sistema de contraseña web denegado; 3) dificultad o error para abrir archivos y links; 4) tener problemas para acceder al correo web.

En el caso del MADGP un 72\% de los alumnos indicó una situación muy parecida, indicando como principales problemas los siguientes: 1) no se podían abrir los archivos; 2) los archivos tienen errores, no es fácil bajar la información; 3) al principio para bajar los contenidos la página se cierra 4) no aceptación de la clave de acceso. 
Mitos y REALIDADES DE LA EDUCACiÓN VIRTUAL (E-LEARNING)...

Estos problemas no pudieron ser solucionados desde las sedes de los Másters, pues en el caso del MEGAP, no existían errores o problemas en la plataforma web por lo que se debía a problemas específicos de los terminales de los alumnos, y en el caso del MADGP fue la propia universidad la que comunicó igual diagnóstico.

Como solución en el caso MADGP se envió parte del material didáctico a través del correo electrónico a fin que los alumnos pudiesen tener acceso a los materiales didácticos (un total de 5 alumnos). En el caso del MEGAP existía prohibición por parte de la coordinación académica del envío del material a través de esta fórmula, por lo que se recurrió finalmente a la coordinación territorial que imprimió el material didáctico y lo entregó personalmente a aquellos alumnos en los que persistía el problema (9 alumnos).

Ante este problema, que no es específico de estos másters, cada vez más autores insisten en la necesidad de realizar páginas web sencillas a efecto de minimizar problemas de conexión debido «al peso» de las mismas, así como evitar volcar videoconferencias y otras herramientas formativas que puedan cargar excesivamente la plataforma del Máster.

\subsection{Ahorro en costes}

Se ha indicado que uno de los factores claves de la expansión del e-learning se debe a que sus costes son sensiblemente inferiores a los de la formación presencial.

Pero en contrapartida, uno de los factores críticos de los Máster on line se relacionaría con el aprendizaje autónomo que deben realizar los alumnos y que lleva a altos índices de deserción (Pisanty, 2003) como consecuencia de la falta de comunicación real entre la organización y el alumno. Por ello, las últimas tendencias en cursos e-learning se centran en un diseño instructivo que mezcle actividades pedagógicas presenciales con actividades on line (denominado como el b-learning) y que pretende subsanar estas carencias motivando al alumno a través de una mayor cercanía.

Si bien ambos másters se concibieron como e-learning puros, no obstante se realizaron actividades presenciales: 5 en el caso del MEGAP (incluyendo inauguración y clausura) y 4 en el caso del MADGP (incluyendo inauguración y clausura).

Estas actividades fueron muy valoradas por parte del alumnado en un $88 \%$ en el caso del MEGAP y en un 95\% en el caso del MADGP. 
Consideramos que la alta valoración de las actividades presenciales se debió a los siguientes aspectos:

1) La posibilidad de poder plantear quejas, sugerencias o problemas de forma interpersonal a los responsables del Máster. 2) Poder resolver dudas con clases teórico-prácticas sobre algunos módulos del Máster a fin de consolidar conocimientos.

Una vez realizadas las actividades presenciales se pudo comprobar, a través de los correos electrónicos enviados por los alumnos, que la motivación de los mismos aumentaba considerablemente al encontrarse más activos en sus comunicaciones.

Obviamente dichas actividades encarecen considerablemente los gastos generales del Máster, pero las consideramos como muy necesarias a efectos de generar una mayor interrelación con los alumnos.

\subsection{Relación entre alumno y profesor}

\subsubsection{Relaciones con los profesores}

Una de las premisas principales para la relación alumno profesor radica en que los profesores tengan un adecuado manejo de las nuevas tecnologías, así como un conocimiento psicopedagógico específico que les permita ponerse en lugar del alumno virtual ( estructura de los términos, rapidez en las respuestas, flexibilidad) a efectos de optimizar el proceso.

Se detectó que un 20\% de los profesores en el caso del MEGAP y un $30 \%$ en caso del MADGP desconocían aspectos esenciales de las nuevas tecnologías que dificultaron su labor docente. Asimismo, también se detectó que la implicación del profesor con los alumnos era sensiblemente inferior que en la enseñanza presencial debido al factor no presencial. Esta situación provocó, en algunos casos, desidia a la hora de responder las dudas de los alumnos o excesiva brevedad en las respuestas, tal como quedó plasmado, con mayor claridad, en el caso del MEGAP donde un 18\% de los tutores no respondieron a las preguntas emitidas por los alumnos.

En términos generales la conclusión obtenida indicó que a mayor edad por parte del profesor, mayores problemas de comunicación existían con los alumnos y un menor interés por las herramientas e-learning.

La comunicación de los alumnos con los profesores se concretó en cuatro aspectos: 
Mitos y REALIDADES DE LA EDUCACiÓN VIRTUAL (E-LEARNING)...

1) Correos electrónicos a consecuencia de las dudas sobre aspectos docentes de las unidades didácticas; 2) comunicación crítica a consecuencia de falta de claridad en los textos debido a la utilización de terminología local, información no actualizada o localista; 3) como consecuencia de la falta de organización del propio alumno, relacionado con su tiempo de estudio y petición de aplazamiento de las evaluaciones y 4) comunicación telefónica específica a consecuencia de explicaciones y desarrollo de conceptos claves no realizables a través de la página web

\subsubsection{Relación con los coordinadores}

En relación a la función de los coordinadores y su relación con los alumnos como facilitadores del proceso de aprendizaje, se siguieron las pautas establecidas por autores como Adell y Sales (1999) o Eisenberg (2005).

Así, las funciones de los coordinadores en ambos Máster se centraron en los siguientes aspectos:1) motivar al alumno; 2) solucionar problemas administrativos con los alumnos; y 3) función académica con el objeto de centrar al alumno en los objetivos del máster.

La motivación fue realizada de manera constante a través de recordatorios sobre las fechas de examen o trabajos, así como una labor personalizada sobre aquellos alumnos en los que se detectó un progresivo abandono de su actividad formativa a través de la comunicación telefónica o vía e-mail.

Si bien no disponemos de datos concretos sobre la influencia directa de esta actividad sobre los alumnos, sí podemos indicar que aquellos alumnos que previamente habían decidido abandonar ambas maestrías, no tuvieron receptividad sobre dicha influencia ni respondieron los correos electrónicos enviados.

En referencia a la solución de problemas administrativos, podemos indicar que esta fue una de las principales labores de la coordinación; según las estadísticas en el caso del MEGAP durante el primer mes se recibieron un total de 62 correos de los cuales 45 se centraron únicamente en aspectos administrativos del máster.

En el último módulo se recibieron un total de 10 mensajes de los cuales 5 eran destinados a problemas en el envío de los módulos, 2 relacionados con aspectos concretos de gestión y 3 enviados a los profesores. 
La media de correos semanales enviados por labores administrativas fue de 8 correos frente a 3 correos relacionados con aspectos docentes.

En le caso del MADGP, durante el primer mes se recibieron un total de 47 correos electrónicos de los cuales 38 eran destinados a cuestiones administrativas. Durante el último módulo del Máster se recibieron un total de 12 correos electrónicos de los cuales todos se centraban en aspectos administrativos.

Como término medio indicar que de los 15 correos semanales que se recibían 13 de los mismos se centraban en materias de gestión.

Respecto a la última labor de los coordinadores (función académica) si bien fue realizada en ambos casos Másters, los alumnos exigían el contacto directo con aquellos profesores que impartían la materia para la solución de problemas y no con los coordinadores, mostrando incluso rechazo sobre este aspecto, tal y como indicaron las encuestas.

En este sentido se comprobó que únicamente los alumnos pedían ayuda a los coordinadores en materia docente cuando se producían los siguientes casos: 1) como intermediación para obtener algo de los profesores; 2) cuando los profesores no habían respondido o no habían solucionado la cuestión planteada.

\section{CONCLUSIONES}

En el trabajo presentado se ha comprobado que ambos máster cumplen tanto las premisas y principios de la educación e-learning como los problemas y errores propios de este tipo de enseñanza. También se han detectado otro tipo de fallos que consideramos extrapolables a distintos cursos de formación e-learning en el campo tratado.

De este modo podemos indicar, desde nuestro punto de vista, cuales podrían ser los motivos que provocan el mayor problema de la educación virtual o e-learning: el alto número de deserciones; pudiendo identificar las siguientes causas:

a) La falta de contacto físico entre alumnos y docentes es un factor más determinante de lo que se ha creído hasta el momento.

b) Los inevitables problemas derivados de las conexiones a Internet en algunos lugares provoca una alta frustración en los alumnos. 
Mitos y REALIDADES DE LA EDUCACión VIRTUAL (E-LEARNING)...

c) Pese al tiempo transcurrido, todavía existe un alto grado de inseguridad ante las nuevas tecnologías por parte de los alumnos.

d) Aplicación de métodos propios de la enseñanza presencial en la enseñanza on line, sin tener en cuenta las diferencias pedagógicas existentes entre ambos tipos de cursos.

e) Dificultad para la explicación de conceptos o teorías específicas de la ciencia política o el derecho a los alumnos a través de los medios de Internet, que obligan a los docentes a la utilización de otras vías de comunicación más tradicionales para la satisfacción del alumnado.

f) A la hora de realizar la selección del alumnado, no se toma en cuenta las características específicas que debe reunir un alumno para realizar un curso de e-learning.

\section{BIBLIOGRAFÍA}

ADECCO. (2009). El estado del arte de la formación». Capital Humano, n. ${ }^{\circ}$ 232. Madrid.

ADELL, J.; SALES, A. (1999). «El profesor online: elementos para la definición de un nuevo rol docente». En línea en: http://www.tecnologiaedu.us.es/edutec/pagina/105 html. (última consulta: 12/09/2009).

ALTVATER, E. (2000). «El lugar y el tiempo de lo político bajo las condiciones de la globalización económica». Zona abierta, n. ${ }^{\circ}$ 92/93, pp. 7-60.

BATES, A. (2001). National strategies for e-learning in post-secundary education and training. Paris: UNESCO.

BERRY, N. (2002). Taking steps towards the knowledge society. Reflections on the process of knowledge development. Luxemburgo: CEDEFOP.

BELLO, M. E. (2003). Educación y globalización, los discursos educativos en Iberoamérica. Barcelona: Anthopos.

BARBERÁ, E. (coord.); BADIA, A.; MOMINÓ, J. M. (2001). La incógnita de la educación a distancia. Barcelona: ICE Universitat Barcelona-Editorial Horsori.

BARBERÁ, E. (2006). "Aportaciones de la tecnología a la e-evaluación». RED Revista de Educación a Distancia. En línea en: http://www.um.es/ead/red/M6 (último acceso: 1/12/2009). 
BARRÓN, H. S. (2004). «Seis problemas de los sistemas universitarios de educación en línea». RED Revista de Educación a Distancia, n. ${ }^{\circ}$ 12. En línea en http://www.um.es/ead/red/barron.pdf (último acceso: 16/10/2009).

BLÁZQUEZ, F.; ALONSO, L. (2005). «Apuntes para la formación del docente de e-learning». Revista enseñanza, n. ${ }^{\circ}$ 23, pp. 65-86: Ediciones Universidad de Salamanca.

CABERO, J.; DUARTE, A. (1999). «Evaluación de medios y materiales de enseñanza en soporte multimedia». Revista de Medios de Educación, n. ${ }^{\circ} 13$, pp. 23-45.

CABERO, J. (2006). «Bases pedagógicas del e-learning». Revista Universitaria y Sociedad del Conocimiento, vol. 3, n. ${ }^{\circ}$ 1. En línea en http:/www.uoc.edu/rusc/3/1dt/esp/cabero.pdf (último acceso: 20/10/2009).

CÁNEPA, C. (2005). «Marco conceptual para la construcción de un modelo de e-learning». Revista de investigación de sistemas de informáticos (RISI). Universidad Nacional Mayor de San Marcos, n. ${ }^{\circ}$ 2 (2), pp. 69-77.

CASTELLS, M. (1996), «La era de la información». Vol. 1. La sociedad en red. Madrid: Alianza Editorial.

CORNELL, I. (1999). «The onrush of technology in education: the professor new dilemma». Educational technology, n. ${ }^{\circ} 39$ (3), pp. 60-64.

CHAMBERS, J. (2001), en Chris Ferry «The work of education in the age of e-college» First Monday, vol. 5, n. ${ }^{\circ}$ 6. En línea en: http://www.firstmonday.org/issue6_5/werry (último acceso: 9/01/2010).

CHIENCHER, A. (2004). "Contextos de aprendizaje presencial y virtual. Algunas características de las interacciones entre profesores y alumnos». En Vogliotti, A.; M. Cortese; M. I. Jacob (comps.), En tiempos de Adversidad: educación pública. Universidad Nacional de Rio Cuatro (Argentina)

CHICKERING, A. W. (1996). "Implementing the seven principles: technology as a lever». American Association of Higer Education Bulletin, n. ${ }^{\circ}$ 10, pp. 3-6.

DAVIES, D. (1998), «The virtual university: a learning university». The Journal of workplace learning, n. ${ }^{\circ} 10$ (4), pp. 175-213.

SCHNECKENBERG, D. (2004). «El e-learning transforma la educación superior». Revista Educar, n. ${ }^{\circ} 33$, pp. 143-156. 
Mitos y REALIDADES DE LA EDUCACiÓN VIRTUAL (E-LEARNING)...

DUTTON, J.; DUTTON, M.; PERRY, J. (2002). «How do online students differ from lectura students?». Journal of Asyncronous Learning, Vol. 6, n. ${ }^{\circ}$ 1. En línea en http://www.aln.org/publications/ jaln/v6n 1/v6n1_dutton.asp (último acceso: 28/11/2009).

DUART, J. M; SANGRÁ, A. (2000). «Formación universitaria por medio de la web: un modelo integrador de enseñanza superior». En A. Duart; J. M. Sangrá (comps.), aprender en la virtualidad. Barcelona: Gedisa.

DRUMMOND, G. (2008). Success in online education: creating a roadmap for student success. Distance learning, vol. 5 iss4, pp. 43-49.

EISENBERG, E. (2005). "The on-line teacher». Artículos de elearningeuropa. Info En línea en:http://www.elearningeuropa. info/index.php?page-doc\&doc_id-6066doclng=6\&menuzone=1 (última consulta: 18/12/2009).

FUNDESCO TELEFORMACIÓN. (1998). Un paso más en el camino de la formación continua. Madrid: Fundesco.

JORBA, J.; SANMARTÍ, N. (1994). Enseñar a aprender y evaluar. Un proceso de evaluación contínua. Barcelona: MEC.

Koskiten, T.; Lacruz, J.; Martinez, M.; Monforte, C.; Montesinos, P. (1999). The great paella cookbook for online learning. Valencia: Centro de Formación de Postgrado-CERES- Universidad Politécnica de Valencia.

LAASER, W. (2006). «El Tema de la calidad en la Educación a Distancia Europea a nivel universitario». RED Revista de Educación a Distancia, n. ${ }^{\circ}$ 16. En línea en http://www.um.es/ead/red/16 (última consulta: 9/12/2009).

MENDEZ-ESTRADA, V.; MONGE NAJERA, J. (2006). «Las TIC en un entorno latinoamericano de educación a distancia: la experiencia de la UNED de Costa Rica. RED. Revista de Educación a Distancia, n. ${ }^{\circ}$ 15. En línea en: http://www.um.es/ead/red/15/ (última consulta: 30/10/2009).

PISANTY, A. (2003). Panorama de la educación a Distancia. En línea en http/:www.edudistan.com/ponencias/Alejandro20\%pisanty\% 20baruch.html (última consulta: 20/01/10).

RUCHLAND, S. \& BREWER, J. (2001) «Implementing on assessment plan to document student learning in a two-year technical college». Journal of vocational education Research, n. ${ }^{\circ} 25$ (2), pp. 141-171. 
ROS, A. (2001). «Risk and opportunities of virtual learning: the experience of UOC». Revista Digital d'Humanitats. En línea en http://www.uoc.edu/humfil/articles/eng/ros/ (última consulta: $17 / 12 / 2009)$

SMITH LYNN, M. (2006). «Best practices in Distance Education». Distance learning Grenwich, vol. 3 Iss 3, pp. 59-67.

ZAPATA, M. (2006). «Distintas formas de intervenir en la distancia y en el elearning. Los modelos de calidad». RED Revista de Educación a Distancia, septiembre, n. ${ }^{\circ} 16$. En línea en: http://www.um.es/ead/red/16 (última consulta: 11/01/2010)

ZIRKIN, B. \& SUMLER, D.(1995). «Interactive or non interactive? That is the question! And annotated bibliography». Journal of Distance Education, n. 10 (1), pp. 95-112. 